\title{
Dynamical phase transition in slowed exclusion processes
}

Tertuliano Franco, Patrícia Gonçalves and Adriana Neumann

\begin{abstract}
In this work, we present symmetric simple exclusion processes with a finite number of bonds whose dynamics is slowed down in order to difficult the passage of particles at those bonds. We study the influence of the rate of passage of mass at those bonds in the macroscopic hydrodynamic equation. As a consequence, we exhibit a dynamical phase transition that goes from smooth profiles to the development of discontinuities.
\end{abstract}

\section{Introduction}

A central question in Statistical Mechanics consists in obtaining the scaling limits of interacting particle systems: given a microscopical interaction in a time-evolving particle system, properly rescaled, what is the limiting behavior of the system? Here, we are concerned about a one-dimensional particle system with a random evolution where the interaction dynamics is given by the exclusion rule, namely, at most one particle can occupy a fixed state (the so-called fermions in Physics) and all the bonds have a constant rate of passage of particles, except a finite number of bonds, whose rate is slowed down in order to difficult the passage across them.

The exclusion rule (Exclusion Process) is a standard model in Probability and Statistical Physics, with wide literature about it. The special bonds

Tertuliano Franco

Universidad de Buenos Aires, Buenos Aires, Argentina e-mail: tertu@impa.br

Patrícia Gonçalves

Centro de Matemática da Universidade do Minho, Campus de Gualtar, 4710-057 Braga, Portugal e-mail: patg@math.uminho.pt

Adriana Neumann

Universidade Federal do Rio Grande do Sul, Porto Alegre, Brasil e-mail: aneumann@impa.br 
slowing down the passage of particles, denominated by slow bonds, have been considered in [3] and have origin in the works [5], [2] and [4].

The scaling limit considered here is the hydrodynamical limit (see [8] for a reference on the subject) for a one-dimensional particle system, where the spatial mesh of the discrete lattice is taken as $N^{-1}$ and particles can evolve at a scaling time given by $N^{2}$. We further assume that at the initial time, the density of particles is approximated by a continuous profile $\gamma(\cdot)$. The parameter characterizing the intensity of the rate of passage at the slow bonds is taken as $N^{-\beta}$, where $\beta \in[0, \infty]$. It is understood here that $N^{-\infty}=0$. In order to keep notation simple we suppose the presence of a single slow bond. The extension to a finite number of slow bonds is straightforward, see [3] for details.

The main result of this paper consists on establishing a dynamical phase transition that depends on the parameter $\beta \in[0, \infty]$, for the hydrodynamic limit of exclusion processes with a finite number of slow bonds. In other words, we prove that, when $N \rightarrow \infty$, the time trajectory of the spatial density of particles converges to a space-time function $\rho(t, x)$ that is the weak solution of a certain partial differential equation, depending on the chosen regime of $\beta$. More precisely, if $\beta \in[0,1)$, then $\rho(t, x)$ is the unique weak solution of the well known heat equation on the torus $\mathbb{T}$ :

$$
\left\{\begin{array}{l}
\partial_{t} \rho=\partial_{x}^{2} \rho \\
\rho(0, \cdot)=\gamma(\cdot)
\end{array}\right.
$$

meaning that, although the rate of passage of particles across the slow bond goes to zero, its microscopical effect is not strong enough in order to have any consequence in the continuum.

On the other hand, at the critical value $\beta=1, \rho(t, x)$ is the unique weak solution of the heat equation on the torus $\mathbb{T}$, with Robin's boundary conditions at the origin:

$$
\left\{\begin{array}{l}
\partial_{t} \rho=\partial_{x}^{2} \rho \\
\rho(0, \cdot)=\gamma(\cdot) \\
\partial_{x} \rho_{t}(1)=\partial_{x} \rho_{t}(0)=\rho_{t}(0)-\rho_{t}(1)
\end{array}\right.
$$

We suppose that the slow bond is close to the origin, otherwise if it is close to some point $u N$ in the one-dimensional discrete torus $\mathbb{T}_{N}$, then the boundary condition is given at $u \in \mathbb{T}$. It is possible to recognize above the Fick's Law (or the Fourier's Law for the heat conduction), which states that the passage of mass across an interface is proportional to the difference of the concentration (or the temperature in the Fourier's Law).

Now, if the rate of passage is slowed down in such a way that $\beta \in(1, \infty]$, then $\rho(t, x)$ is the solution of the heat equation on the torus $\mathbb{T}$, with Neumann's boundary conditions: 


$$
\left\{\begin{array}{l}
\partial_{t} \rho=\partial_{x}^{2} \rho \\
\rho(0, \cdot)=\gamma(\cdot) \\
\partial_{x} \rho_{t}(1)=\partial_{x} \rho_{t}(0)=0
\end{array}\right.
$$

In the case $\beta \in(1, \infty)$, the intensity of the slow bond is big enough in order to prevent the passage of mass in the continuum. In the microscopic scenario, for each fixed $N$, it is possible to observe particles crossing the slow bond, nevertheless in the macroscopic limit, the corresponding boundary is isolated, as predicted by the Neumann's boundary conditions. Microscopically, the case $\beta=\infty$ denotes a forbidden passage of particles across the slow bond and the same behavior is reflected at the continuum, but in this case the system will evolve in a finite box instead of the discrete torus $\mathbb{T}_{N}$.

The paper is organized as follows. In Section 2, we define our microscopic dynamics as an exclusion type model with a finite number of slow bonds whose rate of passage is given by $N^{-\beta}$ with $\beta \in[0, \infty]$. In Section 3 , we present the partial differential equations that we obtain for the different regimes of $\beta$ and we define what we mean by weak solutions of each one of those equations. In Section 4, we formally define the concept of hydrodynamic limit and in Section 5 we state the main result of this paper, namely, the dynamical phase transition at the level of the hydrodynamic limit. We finish the paper, presenting in Section 6, some extensions to higher dimensions and some future open problems.

\section{Microscopic dynamics}

Let $\mathbb{T}_{N}=\mathbb{Z} / N \mathbb{Z}=\{1, \ldots, N\}$ be the one-dimensional discrete torus with $N$ points. We consider a microscopic dynamics of exclusion type, at each site $x \in \mathbb{T}_{N}$ there can be at most one particle. As a consequence of this exclusion rule, our Markov process has state space $\{0,1\}^{\mathbb{T}_{N}}$, its configurations being denoted by the Greek letter $\eta \in\{0,1\}^{\mathbb{T}_{N}}$. The occupation variable at the site $x$ is defined in such a way that $\eta(x)=1$, if the site $x$ is occupied, otherwise $\eta(x)=0$.

Now, we define the dynamics of our interacting particle system. For that purpose, suppose that initially we have particles distributed in $\mathbb{T}_{N}$ as:

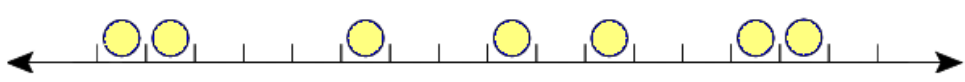

Fig. 1 One possible initial configuration.

Let $p: \mathbb{T}_{N} \times \mathbb{T}_{N} \rightarrow[0,1]$ be a probability measure such that for all $x, y \in \mathbb{T}_{N}$ it holds that $p(x, y):=p(y-x)$. 
At each site $x \in \mathbb{T}_{N}$, there exists a random clock with exponential law of parameter $\lambda(x)$ (usually this parameter is equal to one), which is independent of the random clocks at the other sites. When one clock rings, if there is a particle at that site, then it jumps to a site $y$ with probability $p(y-x)$. By the exclusion rule, particles can only jump to empty sites. For instance, this jump is allowed by the dynamics:

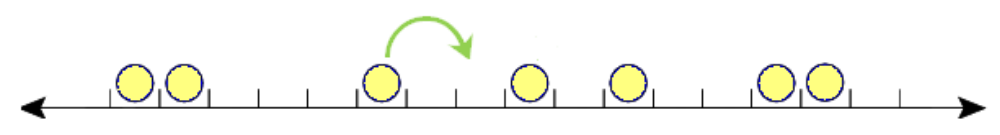

Fig. 2 Since the destination site is empty the particle can jump.

while this other jump is forbidden:

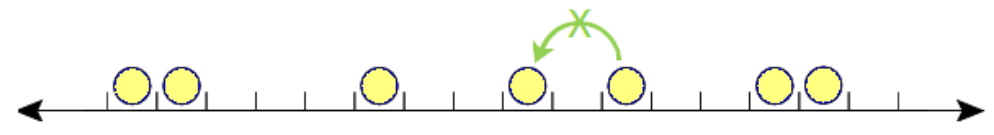

Fig. 3 Since the destination site is occupied the particle does not move.

We suppose that jumps are performed to the nearest-neighbors, namely, two sites $x, y \in \mathbb{T}_{N}$ are nearest-neighbors, that we denote by $x \sim y$, if $|x-y|=$ 1.

We also assume that there are a finite number of slow bonds whose rate of passage of particles is decreased in such a way that those bonds act somehow as a barrier to the movement of particles, see the picture below:

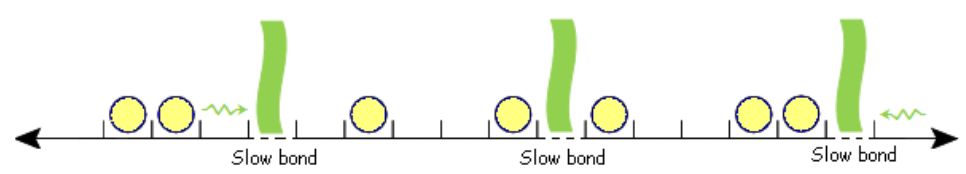

Fig. 4 Exclusion process with three slow bonds acting as a barrier. The rate at the slow bonds is defined in such a way that the passage of particles across them is more difficult than in the other bonds.

To each pair of sites $x, y \in \mathbb{T}_{N}$ such that $x \sim y$, we associate a number $\xi_{x, y}^{N}=\xi_{y, x}^{N}>0$, usually called conductance. At the slow bonds the conductance is a smaller in comparison with the value of the conductance at other 
bonds. The conductances are related to the parameter of the exponential clock as follows: for each site $x \in \mathbb{T}_{N}, \lambda(x)=\sum_{y ; y \sim x} \xi_{x, y}^{N}$.

We consider a finite number of slow bonds, each one associated to a point $b_{1}, \ldots, b_{k} \in \mathbb{T}$. Having a bond associated to the macroscopic point $a \in \mathbb{T}$, means that this bond contains the point $a$ in the natural embedding of the discrete torus $\mathbb{T}_{N}$ in the continuous torus $\mathbb{T}, \frac{1}{N} \mathbb{T}_{N} \subset \mathbb{T}$, see the figure below:

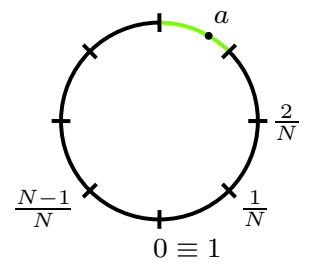

Fig. 5 Discrete torus $\frac{1}{N} \mathbb{T}_{N}$ embedded in continuous torus $\mathbb{T}$. The slow bond is the green one, containing the macroscopical point $a \in \mathbb{T}$.

Given $b_{1}, \ldots, b_{k} \in \mathbb{T}$, we consider the following conductances:

$$
\xi_{x, x+1}^{N}=\left\{\begin{array}{cl}
N^{-\beta}, & \text { if }\left\{b_{1}, \ldots, b_{k}\right\} \cap\left(\frac{x}{N}, \frac{x+1}{N}\right] \neq \varnothing, \\
1, & \text { otherwise }
\end{array}\right.
$$

The rate of the conductances are chosen in such a way that particles cross bonds at rate one, except $k$ particular bonds in which the dynamics is slowed down by a factor $N^{-\beta}$, with $\beta \in(0, \infty]$. Each one of these particular bonds contains the macroscopic point $b_{i} \in \mathbb{T}$; or $b_{i}$ coincides with some vertex $\frac{x}{N}$ and the slow bond is chosen as the bond to the left of $\frac{x}{N}$.

Denote by $\left\{\eta_{t}:=\eta_{t N^{2}}: t \geq 0\right\}$ the Markov process on $\{0,1\}^{\mathbb{T}_{N}}$ whose interacting dynamics is described above, speeded up by $N^{2}$. Although $\eta_{t}$ depends on $N$ and $\beta$, we are not indexing it on that, in order not to overload notation. Formally, this Markov process has generator given on local functions $f:\{0,1\}^{\mathbb{T}_{N}} \rightarrow \mathbb{R}$ by

$$
\mathcal{L}_{N} f(\eta)=\sum_{\substack{x, y \in \mathbb{T}_{N} \\ x \sim y}} \xi_{x, y}^{N}\left(f\left(\eta^{x, y}\right)-f(\eta)\right)
$$

where $\eta^{x, y}$ is the configuration obtained from $\eta$ by exchanging the variables $\eta(x)$ and $\eta(y)$.

Let $D\left(\mathbb{R}_{+},\{0,1\}^{\mathbb{T}_{N}}\right)$ be the path space of càdlàg trajectories with values in $\{0,1\}^{\mathbb{T}_{N}}$. For a measure $\mu^{N}$ on $\{0,1\}^{\mathbb{T}_{N}}$, denote by $\mathbb{P}_{\mu^{N}}^{\beta}$ the probability measure on $D\left(\mathbb{R}_{+},\{0,1\}^{\mathbb{T}_{N}}\right)$ induced by the initial state $\mu^{N}$ and the Markov process $\left\{\eta_{t}: t \geq 0\right\}$. 


\section{Macroscopic hydrodynamic equations}

In this section we present the partial differential equations governing the evolution of the density profile for the different regimes of $\beta$ and we define the notion of weak solutions of each one of these equations.

Denote by $\rho_{t}$ a function $\rho(t, \cdot)$ and for an integer $n$ denote by $C^{n}(\mathbb{T})$ the set of continuous functions from $\mathbb{T}$ to $\mathbb{R}$ and with continuous derivatives of order up to $n$. For $\mathcal{J}$ an interval of $\mathbb{T}$, here and in the sequel we use the notation $C^{n, m}([0, T] \times \mathcal{J})$ for the set of functions defined on the domain $[0, T] \times \mathcal{J}$, that are of class $C^{n}$ in time and $C^{m}$ in space, for $n, m$ integers.

Fix a bounded density profile $\gamma: \mathbb{T} \rightarrow \mathbb{R}$.

Definition 1. A bounded function $\rho:[0, T] \times \mathbb{T} \rightarrow \mathbb{R}$ is said to be a weak solution of the parabolic differential equation:

$$
\left\{\begin{array}{l}
\partial_{t} \rho=\partial_{u}^{2} \rho \\
\rho(0, \cdot)=\gamma(\cdot)
\end{array}\right.
$$

if, for $t \in[0, T]$ and $H \in C^{1,2}([0, T] \times \mathbb{T}), \rho(t, \cdot)$ satisfies the integral equation

$$
\begin{aligned}
& \int_{\mathbb{T}} \rho(t, u) H(t, u) d u-\int_{\mathbb{T}} \gamma(u) H(0, u) d u \\
& -\int_{0}^{t} \int_{\mathbb{T}} \rho(s, u)\left\{\partial_{u}^{2} H(s, u)+\partial_{s} H(s, u)\right\} d u d s=0 .
\end{aligned}
$$

We repeat here the definition of the Sobolev Space from [1].

Definition 2 (Sobolev space). For $a, b \in \mathbb{T}$, the Sobolev space $\mathcal{H}^{1}(a, b)$ consists of all locally summable functions $\zeta:(a, b) \rightarrow \mathbb{R}$ such that there exists $\partial \zeta \in L^{2}(a, b)$ satisfying

$$
\int_{\mathbb{T}} \partial_{u} G(u) \zeta(u) d u=-\int_{\mathbb{T}} G(u) \partial \zeta(u) d u,
$$

for all $G \in C^{\infty}(a, b)$ with compact support. For $\zeta \in \mathcal{H}^{1}(a, b)$, we define the norm

$$
\|\zeta\|_{\mathcal{H}^{1}(a, b)}=\|\partial \zeta\|_{L^{2}(a, b)} .
$$

Definition 3. The space $L^{2}\left(0, T ; \mathcal{H}^{1}(a, b)\right)$ consists of all measurable functions $\xi:[0, T] \rightarrow \mathcal{H}^{1}(a, b)$ with

$$
\|\xi\|_{L^{2}\left(0, T ; \mathcal{H}^{1}(a, b)\right)}:=\left(\int_{0}^{T}\left\|\xi_{t}\right\|_{\mathcal{H}^{1}(a, b)}^{2} d t\right)^{1 / 2}<\infty .
$$

Definition 4. Let $\left\{b_{1}, \ldots, b_{k}\right\} \subset \mathbb{T}$. A bounded function $\rho:[0, T] \times \mathbb{T} \rightarrow \mathbb{R}$ is said to be a weak solution of the following parabolic differential equation with Robin's boundary conditions at the points $\left\{b_{1}, \ldots, b_{k}\right\} \subset \mathbb{T}$ : 


$$
\left\{\begin{array}{l}
\partial_{t} \rho=\partial_{u}^{2} \rho \\
\rho(0, \cdot)=\gamma(\cdot) \\
\partial_{u} \rho_{t}\left(b_{i}^{+}\right)=\partial_{u} \rho_{t}\left(b_{i}^{-}\right)=\rho_{t}\left(b_{i}^{+}\right)-\rho_{t}\left(b_{i}^{-}\right), \forall t \in[0, T], \forall i=1, \ldots, k
\end{array}\right.
$$

if the following two conditions are fulfilled:

(1) $\rho \in L^{2}\left(0, T ; \mathcal{H}^{1}\left(\mathbb{T} \backslash\left\{b_{1}, \ldots, b_{k}\right\}\right)\right)$;

(2) For all functions $H \in C^{1,2}\left([0, T] \times \mathbb{T} \backslash\left\{b_{1}, \ldots, b_{k}\right\}\right)$ and for all $t \in[0, T]$, $\rho(t, \cdot)$ satisfies the integral equation

$$
\begin{aligned}
& \int_{\mathbb{T}} \rho(t, u) H(t, u) d u-\int_{\mathbb{T}} \gamma(u) H(0, u) d u \\
- & \int_{0}^{t} \int_{\mathbb{T}} \rho(s, u)\left\{\partial_{u}^{2} H(s, u)+\partial_{s} H(s, u)\right\} d u d s \\
- & \sum_{i=1}^{k} \int_{0}^{t}\left\{\rho\left(s, b_{i}^{+}\right) \partial_{u} H\left(s, b_{i}^{+}\right)-\rho\left(s, b_{i}^{-}\right) \partial_{u} H\left(s, b_{i}^{-}\right)\right\} d s \\
+ & \sum_{i=1}^{k} \int_{0}^{t}\left\{\rho\left(s, b_{i}^{+}\right)-\rho\left(s, b_{i}^{-}\right)\right\}\left\{H\left(s, b_{i}^{+}\right)-H\left(s, b_{i}^{-}\right)\right\} d s=0 .
\end{aligned}
$$

Definition 5. Let $\left\{b_{1}, \ldots, b_{k}\right\} \subset \mathbb{T}$. A bounded function $\rho:[0, T] \times \mathbb{T} \rightarrow \mathbb{R}$ is said to be a weak solution of the following parabolic differential equation with Neumann's boundary conditions at the points $\left\{b_{1}, \ldots, b_{k}\right\} \subset \mathbb{T}$ :

$$
\left\{\begin{array}{l}
\partial_{t} \rho=\partial_{u}^{2} \rho \\
\rho(0, \cdot)=\gamma(\cdot) \\
\partial_{u} \rho\left(t, b_{i}^{-}\right)=\partial_{u} \rho\left(t, b_{i}^{+}\right)=0, \forall t \in[0, T], \forall i=1, \ldots, k
\end{array}\right.
$$

if the following two conditions are fulfilled:

(1) $\rho \in L^{2}\left(0, T ; \mathcal{H}^{1}\left(\mathbb{T} \backslash\left\{b_{1}, \ldots, b_{k}\right\}\right)\right)$;

(2) For all functions $H \in C^{1,2}\left([0, T] \times \mathbb{T} \backslash\left\{b_{1}, \ldots, b_{k}\right\}\right)$ and for all $t \in[0, T]$, $\rho(t, \cdot)$ satisfies the integral equation

$$
\begin{aligned}
& \int_{\mathbb{T}} \rho(t, u) H(t, u) d u-\int_{\mathbb{T}} \gamma(u) H(0, u) d u \\
- & \int_{0}^{t} \int_{\mathbb{T}} \rho(s, u)\left\{\partial_{u}^{2} H(s, u)+\partial_{s} H(s, u)\right\} d u d s \\
- & \sum_{i=1}^{k} \int_{0}^{t}\left\{\rho\left(s, b_{i}^{+}\right) \partial_{u} H\left(s, b_{i}^{+}\right)-\rho\left(s, b_{i}^{-}\right) \partial_{u} H\left(s, b_{i}^{-}\right)\right\} d s=0 .
\end{aligned}
$$

For classical results about Sobolev spaces, we refer the reader to [1] and [9]. Since in Definition 4 and 5 we imposed $\rho \in L^{2}\left(0, T ; \mathcal{H}^{1}\left(\mathbb{T} \backslash\left\{b_{1}, \ldots, b_{k}\right\}\right)\right)$, the integrals above are well-defined at the boundary points.

Heuristically, in order to establish an integral equation for the weak solution of the heat equation with Robin's or Neumann's boundary conditions 
as above, one should multiply both sides of (2) or (4) (respectively) by a test function $H$, integrate in space and time and then perform twice a formal integration by parts, obtaining the equation

$$
\begin{aligned}
& \int_{\mathbb{T}} \rho(t, u) H(t, u) d u-\int_{\mathbb{T}} \gamma(u) H(0, u) d u \\
- & \int_{0}^{t} \int_{\mathbb{T}} \rho(s, u)\left\{\partial_{u}^{2} H(s, u)+\partial_{s} H(s, u)\right\} d u d s \\
- & \sum_{i=1}^{k} \int_{0}^{t}\left\{\rho\left(s, b_{i}^{+}\right) \partial_{u} H\left(s, b_{i}^{+}\right)-\rho\left(s, b_{i}^{-}\right) \partial_{u} H\left(s, b_{i}^{-}\right)\right\} d s \\
+ & \sum_{i=1}^{k} \int_{0}^{t}\left\{\partial_{u} \rho\left(s, b_{i}^{+}\right) H\left(s, b_{i}^{+}\right)-\partial_{u} \rho\left(s, b_{i}^{-}\right) H\left(s, b_{i}^{-}\right)\right\} d s=0 .
\end{aligned}
$$

Applying the formal boundary conditions on $\rho$, one gets to (3) or (5), respectively. Besides that, any strong solution of (2) or (4) is a weak solution of (2) or (4), respectively.

\section{Hydrodynamic Limit}

In this section we define formally the hydrodynamic limit for the processes we described above. For that purpose, we define the empirical measure by:

$$
\pi_{t}^{N}(d u)=\pi^{N}\left(\eta_{t}, d u\right)=\frac{1}{N} \sum_{x \in \mathbb{T}_{N}} \eta_{t}(x) \delta_{\frac{x}{N}}(d u),
$$

where $\delta_{u}$ denotes the Dirac measure at $u \in \mathbb{T}$. As mentioned in the introduction we assume that at the initial time the density of particles is approximated by a given profile. Now we define exactly what is the assumption we need on the initial distribution of the system.

Fix a continuous density profile $\gamma: \mathbb{T} \rightarrow[0,1]$ and denote by $\left(\mu^{N}\right)_{N}$ a sequence of probability measures on $\{0,1\}^{\mathbb{T}_{N}}$.

Definition 6. A sequence $\left(\mu^{N}\right)_{N}$ is associated to an initial profile $\gamma(\cdot)$, if for every continuous function $H: \mathbb{T} \rightarrow \mathbb{R}$ and for every $\delta>0$

$$
\lim _{N \rightarrow+\infty} \mu^{N}\left[\left|\frac{1}{N} \sum_{x \in \mathbb{T}_{N}} H\left(\frac{x}{N}\right) \eta(x)-\int_{\mathbb{T}} H(u) \gamma(u) d u\right|>\delta\right]=0 .
$$

We can translate the definition above by saying that a sequence of measures $\left(\mu^{N}\right)_{N}$ is associated to a profile $\gamma(\cdot)$ if a Law of Large Number (in the weak sense) holds for the empirical measure at time $t=0$ under the probability $\mu^{N}$. We can rewrite (6) as 


$$
\lim _{N \rightarrow+\infty} \mu^{N}\left[\left|\int_{\mathbb{T}} H(u) \pi_{0}^{N}(d u)-\int_{\mathbb{T}} H(u) \gamma(u) d u\right|>\delta\right]=0 .
$$

The goal in hydrodynamic limit consists in showing that if at time $t=0$ the empirical measures are associated to some initial profile $\gamma(\cdot)$, then at time $t$ they are associated to a profile $\rho_{t}$, where $\rho_{t}$ is the solution of the corresponding hydrodynamic equation. So, the goal is to show that if a Law of Large Numbers holds for the empirical measure at time $t=0$ then it holds at any time $t$.

In order to prove this result, we follow the Entropy Method which was introduced by Guo, Papanicolau and Varadhan in [7]. This method requires the uniqueness of weak solutions of the hydrodynamic equation. We notice that all the partial differential equations (1), (2) and (4), have a unique weak solution. For details we refer the reader to [3].

\section{The main result}

Here, we state the main result of the article in which we establish the hydrodynamic limit for the exclusion process with slow bonds depending on the regime of $\beta$ :

Theorem 1. (Franco, Gonçalves and Neumann [3])

Fix $\beta \in[0, \infty]$. Consider the exclusion process with $k$ slow bonds corresponding to macroscopic points $b_{1}, \ldots, b_{k} \in \mathbb{T}$ and with conductance $N^{-\beta}$ at each one of these bonds. Fix a continuous profile $\gamma: \mathbb{T} \rightarrow[0,1]$. Let $\left(\mu^{N}\right)_{N}$ be a sequence of probability measures on $\{0,1\}^{\mathbb{T}_{N}}$ associated to $\gamma(\cdot)$.

Then, for any $t \in[0, T]$, for every $\delta>0$ and every $H \in C(\mathbb{T})$, it holds that

$$
\lim _{N \rightarrow \infty} \mathbb{P}_{\mu^{N}}^{\beta}\left\{\eta .:\left|\frac{1}{N} \sum_{x \in \mathbb{T}_{N}} H\left(\frac{x}{N}\right) \eta_{t}(x)-\int_{\mathbb{T}} H(u) \rho(t, u) d u\right|>\delta\right\}=0,
$$

where :

- if $\beta \in[0,1), \rho(t, \cdot)$ is the unique weak solution of $(1)$;

- if $\beta=1, \rho(t, \cdot)$ is the unique weak solution of $(2)$;

- if $\beta \in(1, \infty], \rho(t, \cdot)$ is the unique weak solution of $(4)$.

\section{Future problems}

We finish the paper by presenting some extensions to higher dimensions and some future problems.

The extension of the results presented here to higher dimensional cases can be obtained as in [5]. In that paper the slow bonds have a spatial position 
associated to a smooth closed surface, modeling a membrane slowing down the passage of particles. There, the authors study the case $\beta=1$ and it is an interesting problem to obtain the dynamical phase transition as in [3], for any $d$-dimensional exclusion process with slow bonds. Also, it would be a challenging problem to extend the result to particle systems without exclusion constrains, as for example, the generalized exclusion or the zero-range process.

One interesting problem that we are studying is related to the dynamical phase transition at the level of the fluctuations or at the limit distributions of a tagged particle. In the former case, we fix a profile $\gamma(\cdot)$ and we suppose the system to start from the Bernoulli product measure $\nu_{\gamma(\cdot)}$, with parameter $\gamma(x / N)$ at the site $x \in \mathbb{T}_{N}$. We consider the density fluctuation field defined as:

$$
y_{t}^{N}:=\frac{1}{\sqrt{N}} \sum_{x \in \mathbb{T}_{N}} \delta_{x / N}\left\{\eta_{t}(x)-\mathbb{E}_{\nu_{\gamma(\cdot)}}\left[\eta_{t}(x)\right]\right\} .
$$

The goal is to obtain the limit $y$. of the density field as defined above and to characterize it as a solution to some stochastic partial differential equation. As in the hydrodynamic limit scenario, this equation will depend on the regime of $\beta$. For $\beta=0$, it was proved in [10] that $y$. is an Ornstein-Uhlenbeck process with certain characteristics depending on the non-equilibrium thermodynamical quantities of the underlying system. The goal is to characterize the limit process for $\beta \neq 0$. In the case of the tagged particle, at the initial time we fix one of the particles that are distributed according to $\nu_{\gamma(\cdot)}$ and we follow its trajectory. We want to establish a dynamical phase transition at the level of the limiting distributions of this particle. For $\beta=0$, it is proved in [6] that the limit distributions are given by a fractional Brownian motion. We want to characterize the limit when $\beta \neq 0$. This is a step towards characterizing the limit behavior of exclusion processes with slow bonds.

\section{References}

1. Evans, L. (1998): Partial Differential Equations. [Graduate Studies in Mathematics], American Mathematical Society.

2. Faggionato, A.; Jara, M.; Landim, C. (2008): Hydrodynamic behavior of one dimensional subdiffusive exclusion processes with random conductances, Probability Theory and Related Fields, 144, no. 3-4, 633-667.

3. Franco, T.; Gonçalves, P.; Neumann, A.: Hydrodynamical behavior of symmetric exclusion with slow bonds, to appear in Annales de l'Institut Henri Poincaré: Probability and Statistics (B).

4. Franco, T.; Landim, C. (2010): Hydrodynamic Limit of Gradient Exclusion Processes with Conductances, Archive for Rational Mechanics and Analysis, 195, 409-439.

5. Franco, T.; Neumann, A.; Valle, G. (2011): Hydrodynamic Limit for a Class of Exclusion Type Process in Dimension $\geq 2$, Journal of Applied Probability, 49.2, 333-351.

6. Jara, M., Landim, C. (2006): Non Equilibrium Central Limit Theorem for a Tagged Particle in Symmetric Simple Exclusion, Annales de l'Institute Henri Poincaré: Probability and Statistics, 42, no. 5, 567-577. 
7. Guo, M. Z.; Papanicolau, G. C.; Varadhan, S. R. S. (1988): Nonlinear diffusion limit for a system with nearest neighbor interactions, Communications Mathematical Physics, 118, 31-59.

8. Kipnis, C.; Landim, C. (1999): Scaling Limits of Interacting Particle Systems, Springer-Verlag, New York.

9. Leoni, G. (2009): A First Course in Sobolev Spaces, [Graduate Studies in Mathematics], American Mathematical Society.

10. Ravishankar, K. (1992): Fluctuations from the hydrodynamical limit for the symmetric simple exclusion in $\mathbb{Z}^{d}$, Stochastic Processes and their Applications, 42, 31-37. 